\title{
loT-assisted physical education training network virtualization and resource management using a deep reinforcement learning system
}

\author{
Qiang $\mathrm{Li}^{1} \cdot$ PriyanMalarvizhi Kumar ${ }^{2} \cdot$ Mamoun Alazab ${ }^{3}$
}

Received: 15 July 2021 / Accepted: 4 November 2021 / Published online: 2 December 2021

(c) The Author(s) 2021

\begin{abstract}
The Internet of Things (IoT) development made it possible for technology to communicate physical education by connecting cost-effective heterogeneous devices and digital applications to uncontrolled and accessible environments. The traditional physical education monitoring environment creates crucial manual efforts on athletes' activity observations and tracking consistently. Similarly, remote monitoring and assessment of athletes in sports training seem to be barriers to physical education monitoring and training. It creates various chances to improve training and education through technology advancements like IoT and deep learning. Students can efficiently monitor their physical behavior to increase their physical and psychological benefits. The IoT-assisted physical activity monitoring device is proposed to track students' physical activity and enhance outcomes. The management ability allows students to organize and increase speed their physical activity in a wellness manner. In addition, this study examines the connections between monitoring ability which is an essential component for sports activities and physical activity. This system collects essential information from IoT-based wearable devices that interact with the data in real time by virtualizing the device. The IoT network includes several device activities and monitors the heartbeat and physical body temperature of a person. The analysis of specific studies and student feedback shows that the designed virtual system of physical educations is effective in its application and implementation and provides a reliable guide for developing student physical educational systems. The experimental analysis is evaluated; the solution offered is developing and supporting physical education and training approaches in reality and creates healthy environment systems to solve the health monitoring challenges posed by IoT devices. The proposed method has achieved extraordinary physical activity monitoring compared to the conventional systems, as shown by experimental findings. The simulation analysis of physical education can help students and improve the associated aspects of physical abilities with high accuracy ratio (98.3), prediction ratio $(96.5 \%)$, interaction ratio $(94.4 \%)$, performance ratio $(95.1 \%)$, the efficiency ratio $(93.2), F$-score $(92.2 \%)$, and reduce error rate $(17.5 \%)$ and physical activity patterns.
\end{abstract}

Keywords Internet of Things (IoT) · Physical activity $\cdot$ Physical education $\cdot$ Students $\cdot$ Wearable device

Qiang Li

lq18070038978@sina.com

PriyanMalarvizhi Kumar

P.Malarvizhikumar@ieee.org

Mamoun Alazab

m.alazab@icsl.com.au

1 Department of Physical Education, Jiangxi University of Finance and Economics, Nanchang 330013, China

2 Department of Computer Science and Engineering, Kyung Hee University, Seoul, South Korea

3 IT and Environment, Charles Darwin University, Casuarina, Australia

\section{Significance of loT-assisted physical education training network virtualization and resource management}

The training effect of physical education is an important aspect of the educational sector and is directly linked to progress in national education and health [1]. Physical activity and thus physical education help pupils build competence and self-confidence. Sports at schools and colleges assist students in acquiring a wide variety of abilities and teaching them techniques, methods, and newer ideas for success at home and school. This reason is why physical education programs are so important at schools and colleges. Several 
challenges in training experience such as lack of detailed analyses of virtual training analytical activity are shown by current data [2]. The overall growth has been demonstrated that students constantly demand the substance and form of physical education [3]. Physical education at educational institutions does not fulfill the standards influencing the increasing change of education [4]. Physical education approaches that correspond to training at academic institutions and improve student effectiveness are highly significant to analyze [5]. It offers a possibility to create physical education by developing virtual network technology, the Internet of Things (IoT), and wearable device advanced information technology [6]. IoT and wearable technology in sports introduce a new era in athlete training, mostly for performance monitoring/evaluation and fitness assessment. To do this, IoT wearable technologies are typically employed, which rely on sensor systems. It gathers, analyzes, and sends information such as biomarkers or other relevant indications that can be used to assess the athlete's capability and evaluate its physical and health problems, therefore enhancing its performance levels. To measure athletic performance, virtual networks are utilized as training aids in various sports such as golf, athletics, skiing, cycling, etc.

The current development of physical activities in educational institutes using environments and resources is ineffective [7]. They unaware that their physical activity and extracurricular activity management cannot provide actual student needs [8]. Basic principles of management are by the different practices of the students [9]. Physical activities are conducted in educational establishments are largely alone and analytically insufficient [10]. The IoT gives various opportunities to improve education and training using deep reinforcement learning [11]. Combining multiple applications of deep reinforcement learning techniques with IoT data and wearable physical analytics can accurately monitor each student's physical condition [12]. As part of an IoT alliance, cloud computing stores data from IoT devices in the cloud. In the cloud, computer resources are stored on a centralized server that can be accessed anytime needed. The IoT generates sensor data, and cloud computing provides a convenient way to transport and store that data. Infrastructures based on Internet cloud computing enable IoT to make sense of the vast quantity of data created. If users want more or less storage, they may do it without any concern. As the volume of data created grows, they can simply scale up their storage and pay only for what they need with Internet cloud computing.

Deep reinforcement learning enables customized education in flexible physical activities [13]. The new economic, physical education system is based on modern information technologies, such as deep learning and IoT [14]. Its core is students' training and physical education, the provision of technical strategies, and the particular qualities of physical activities [15]. For deep reinforcement learning to work in sports training, incentives and penalties are used to educate a decision-making agent on making optimum judgments. By delivering feedback on actions that lead to specific results, it aims to mimic how we learn as athletes via trial and error. Reinforcement learning frameworks can be tested using realtime sports monitoring, since they generally give a clear positive or negative outcome that can be utilized as the intended reward signal.

Physical education aims to increase students' physical experiences and abilities, well-being, and the capacity to use it in a wide range of physical activities [16]. Physical education is usually defined as an organization of sports and physical training [17]. All these interactions together provide an essential understanding of the physical education concept [18]. Physical education focuses on physical conditioning and the ability to do and appreciate physical exercise [19]. An effective study in physical education should include instruction, student, emotional health and trainers, quality education, and a student assessment [20]. Physical training helps students to build confidence and physical abilities. For example, the education offers students activities to identify and improve their skills, such as running, catching, throwing, etc. [21]. Physical education develops strength and improves the ability to participate for a lifetime in physical activity. Physical education programs promote health advantages and disadvantages of daily exercise and balanced healthy intake in the dysfunction and poor diet $[22,23]$. It improves the psychological and community abilities of the students and contributes positively to their physical, social, emotional, and psychological growth [24]. Physical exercise can thus be used as a sector not concentrated on physical conditioning to develop a range of competencies and abilities. The physical activity teaches collaboration, respect, integrity, confidence, growth in grace, and the loss of hope. Assuming that physical activity constantly reduces with age, the average level and the variation of physical challenges are assumed to rise with increased physical education. In short, the results show that physical activity motivation can improve physical capabilities for regular exercise and increase physical conditioning. This study gives opportunities for supporting IoT-based education in the sectors of students and behavioral health development and recovery through the described methodology, as especially established to monitor physical control abilities.

The main contribution of the paper is:

- Designing the IoT-PAMD is proposed for monitoring and enhancing students' physical fitness.

- This study explores interactions that are important to sports activities and physical activity between monitoring capabilities. 
- This system collects critical data from wearable IoT-based devices and communicates with data in real time by virtualization.

- The experimental analysis evaluates the solution that offers to develop and support physical education and training systems and promote effective environment systems to solve the challenges posed by IoT devices for health monitoring.

The remaining structure of the paper is organized as follows: "Literature work" explores the related work and its corresponding discussion, and "The Internet of Things-assisted physical activity monitoring" details the proposed IoT-PDMD for monitoring the physical activity. In "Results and discussion", results and discussion have been compared with an existing method. "Conclusion" concludes the paper based on the previous section's analysis and discussion.

\section{Literature work}

Children who participate in physical activities in and out of school should get the skills and confidence to participate in a wide range of physical activities. All children can enjoy and achieve various physical activities with the help of a high-quality PE program that incorporates technology. This section summarizes the comprehensive literature study with the most significant research on physical education monitoring and training using IoT and machine intelligence.

An artificial neural network (ANN) based on accelerometer data processing was presented by Du and Nan [25]. As part of the audit, accelerometer-based wearable movement identifiers were used to measure real actions, such as postural and movement arrangement, energy consumption, fall detection, and assessment of balance control evaluations. There were comparisons between the FPGA tool's current development status and the entire perspective of potential technologies. They gave a reliable guideline for further reform of college physical activities.

Haible et al. [26] determined that persons have to effectively control their physical activity to increase the associated health benefits. Controlling ability enables the person to improve health structure and pace physical activity. The concept was established in a framework of health skills related to physical activity, and is connected to health literacy and physical literacy. Therefore, this study focused on establishing a self-reporting scale to measure students' physical and psychological aspects of control skills. The second issue analyzed the interaction between control experience and its basic elements, information, motivation, control ability, sport, and physical conditioning. In addition, the data supported the importance of maintaining skills to gain health benefits through physical activity.
Zhong et al. [3] introduced the system that continuously monitors safety culture using different sensors associated with the physical activity recognition and monitoring model (PARMM), regarded as a major paradigm of smart health care. This research developed a secure, energy-efficient student monitoring system capable of providing health data in real-time studies. This could enable students to monitor students' health statistics for temperature, heartbeat, pressure, and body temperature in real time. This paper extended PARMM to developing IoT (IoT) technology by integrating diverse economically efficient wearable gadgets to open environments. The experimental study was validated to solve the new issues of the IoT environment for effective health monitoring for college students according to PARMM systems.

Telford et al. [27] aimed to assess the implementation, acceptance, and impact of physical education (PE) distribution as part of the physical education and physical literacy approach (PEPLA) designed to improve teachers' physical education and physical literacy opportunities in urban educational institutions. The role of the trainer was to support teachers and develop professionally in physical education and develop chances for physical literacy in and out of the school environment. The PEPL trainer involved frequent physical activity before and after, schools and a training system for sports activities. PEPL was well received by teachers and management, and the teachers' approach led to physical education being introduced in their weekly education curriculum and external sport training programs.

Jalal et al. [28] expressed the new human activity recognition system (HARS) to monitor physical activities from continuous sequences by inertial sensors. In particular, they reported on wearable sensors that continuously monitor human health by a range of positions and behaviors to provide people with effective and timely care. The proposed HARS filtered the one-dimensional signal using a brief filter that checks the low and upper frequencies for optimal sensor data processing. The suggested system was applied and evaluated on three challenges for the model's viability to be assessed.

Lee et al. [29] demonstrated that the attitude and perspective of older people were linked to their participation in leisure-time physical activity (LTPA) to provide insight into physical activity promotion. Multiple surveys have been used to evaluate the attitudes and perspectives of elderly persons in different fields. The associations between predictors and outcome variables were examined through a technique for adjusted multivariate. Psychological variables were connected substantially with older ones LTPA, such as selfefficacy, intrinsic motivation, spiritual involvement, social connections, and neighborhood safety. In contrast, neighborhoods' perceived social relations and safety measures showed varying walking and sports and exercise relationships with their intensity. 
Based on extensive data analysis, QI-QI Zhang [30] developed an intelligent control mechanism for sports players' training progress. Through the construction of the evaluation system, the intelligent control algorithm of sports athletes' training intensity was improved, and the operating effectiveness of the intelligent control model of sports athletes' training intensity was ensured. Simulation tests were carried out to check the model's energy, and the findings demonstrate that most models are effective. Analysis-wise, the intelligent model of athletes' training development is beneficial for our research extension.

According to a recent study review, students with practical backgrounds can benefit from motion tracking systems by determining where they are physically located. Consequently, these models can rapidly and effectively construct training plans, address the data fluctuation issue under the trend of big data division, and achieve intelligent operation, among other things. This research implements deep reinforcement learning to ensure accurate data analytics with minimal processing stages and complexities. Based on the survey, accuracy ratio, prediction ratio, performance ratio, efficiency ratio, interaction ratio, $F$-score, and error rate in existing PARMM, PEPLA, HARS, and LTPA methods.

\section{The Internet of Things-assisted physical activity monitoring}

The physical activity and resource management based on an IoT system collects student physical data on wearable sensing devices with accessibility and protected intermediate systems. The wearable devices can collect information on physical health, such as weight, sleep, heart rate, and blood pressure in a student's environment. Due to the technical and operational range of accelerometers and GPS microelectric systems, personal assistance devices are frequently used for monitoring and access by normal students of certain low-level physical types. The virtualization network is generally supported with wired and wireless connections such as Bluetooth and wireless Internet. Physical activity monitoring systems have been used for students assessed healthy data under controlled conditions such as improving the quality of a living and replicated activity sequencing. The technique is analyzed by wearable devices to monitor the biomedical parameters of students during their everyday physical activities. Wearable devices have become more desirable without assisting in everyday student physical activities to monitor. Such devices can be more physiological signals, as their activity and development are calculated by positioning them within the body in several places. Improved competitive correspondence innovation, economical processing, and low service associated with IoT wearable devices have prepared the way for minimum effort and orderly long- range physical health monitoring. This data collection aims to provide students with essential information for identifying physical activity and monitoring the involvement of healthy students in the data collection process. The physical activity evaluation aims to determine the frequency, length, intensity, and types of student behavior. Monitoring heart rate by accelerometers and wearable devices are all standardized approaches for physical activity evaluations. Physical activity assessment provides vital information on an individual's fitness regarding specified health standards, awareness-raising of students, and physical activity requirements monitoring. The purpose of the IoT-assisted physical activity monitoring device is to promote the healthy environment of students. The proposed approach uses IoT and wearable wireless devices for student physical activity data analysis.

Figure 1 shows the components of physical education. This paper found that similar difficulties can be seen in three principal components: physical education games, dance, and gymnastics. Professional trainers at educational institutions often focus on physical activity that students usually enjoy and can easily be taken into account to deal with their perceived obstacles in physical education without a high level of specific content experience. Physical training has been critically analyzed to bring together more education that can be defined strength and student physical fitness. The effects of physical education for students can be increasingly recognized and the reality of health. It is considered that more direct teaching of skills and physical conditioning is more effective than physical training to increase the chances of students taking part in organized sport and physical activity. This concentration on abilities and physical activity goes against play games that take the immediate interests of students for many specialist trainers in the classroom. Sports have more personal significance in skills and physical training sessions, and sports typically create higher passion and participation.

The separation of the 'field' of physical education is another challenge for a specialist teacher at an educational institution. In contrast to a training and fitness session, a sport is essentially a social event, where the need to achieve as an individual is the main motivation. These individually focused situations can often isolate students from positioning themselves in a successful. However, the present paper is concerned that the existing training method addresses the student's needs and provides conditions for students to participate in effective physical activity. Such activities should not come from requests for evaluation and reporting to evaluate students against more general data sources.

The focus of their activities is a significant difference between these three positions. Physical training in large open spaces such as the sports field and the playground usually occurs. In less formal areas such as the park, outdoor train- 
Fig. 1 The components of physical education

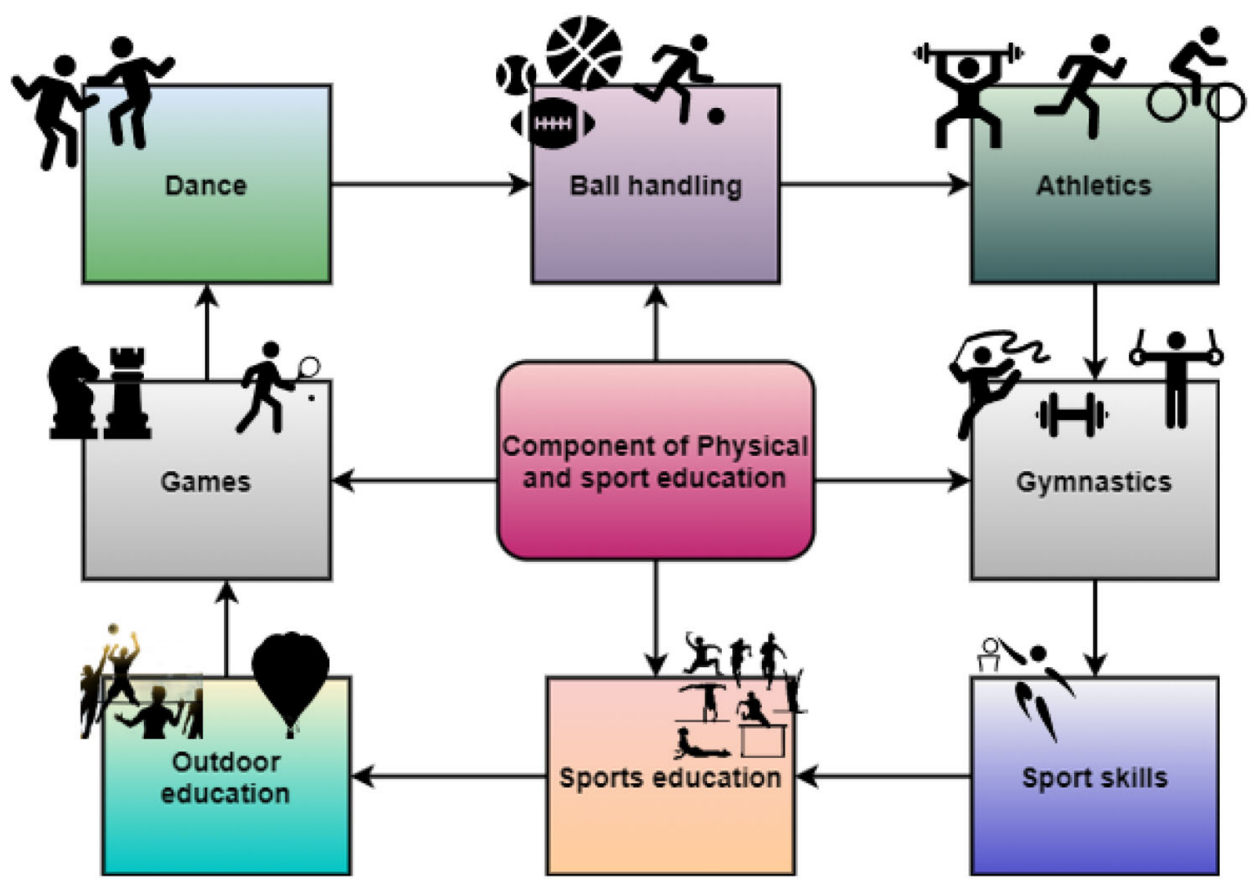

ing often takes a step further. Physical education is typically given out in a classroom environment. Each of these sections covers a different technique to connect students to the educational information. Each implies another interaction that is most prevalent in the present teaching approaches between students and curriculum. Succession describes the relation between health, physical education, and outdoor physical training. Figure 1 shows an end to this continuum of health education intended to give back specific knowledge related to health education provided by the curriculum. Students can remember these facts when necessary and it is assumed that students can transfer their knowledge of the classroom to other housing conditions. The assumption of knowledge directly leading to positive abnormal behavior changes is difficult for most trainers. The effective process for physical education is to establish supportive education institutions, community policies, and practices to enhance what can be learned in education. However, the physical education form centered on a classroom embraces a teaching approach motivated by the fundamental and relatively abstract understanding that starts from the curriculum directly and aims to give students that. Although there are still desires and results to be achieved, the student's interests are answered explicitly in the teacher's thoughts. The trainer aims to define a relatively long-term scenario that is responsive to the students' attentiveness, usually without any specific reference to the formality of the curriculum contents and can properly use the potential of the external discussion on physical education. There are results meant for this experience shared language of a formal curriculum that often combines abstract data are often difficult to analyze. Physical education appears to be partitioning the curriculum between every end including by student's interests during sports, often in connection with the more education process, as in physical activity training.

Although personal and social components are considered important elements in physical education, instructional approaches usually relegate many of these components to classroom management. Physical education should be performed by responsive presenting rather than by a determined effort to understand coherent ideas. However, several of the student's interests related to physical education can be found within these personal and social components. In systematic physical training, skill and fitness components can take a student-appropriate position as a developing focus instead of an emotion beginning with these personal and social elements.

Figure 2 illustrates the physical activity and resource management with competent care monitoring. The IoT fourlayer physical activity resource management framework uses interoperability and security protocols to collect personal data from various wearable sensor devices. Wearable devices such as sleep, weights, heart rate, blood pressure, and student background can collect physical health data. As accelerometer technology and GPS microelectromechanical system technological and operational maturity are technically operating, ordinary students widely use personal assistance devices to monitor and access certain low-level physical activity types. The network is usually wired and wirelessly supported such as Bluetooth and Wi-Fi networks. The data typically contain redundancy to be extracted and transmitted in an under classified data layer from preprocessing to event form as indicated in Fig. 2. An IoT gateway 
Fig. 2 Physical activity and resource management with smart care monitoring analysis

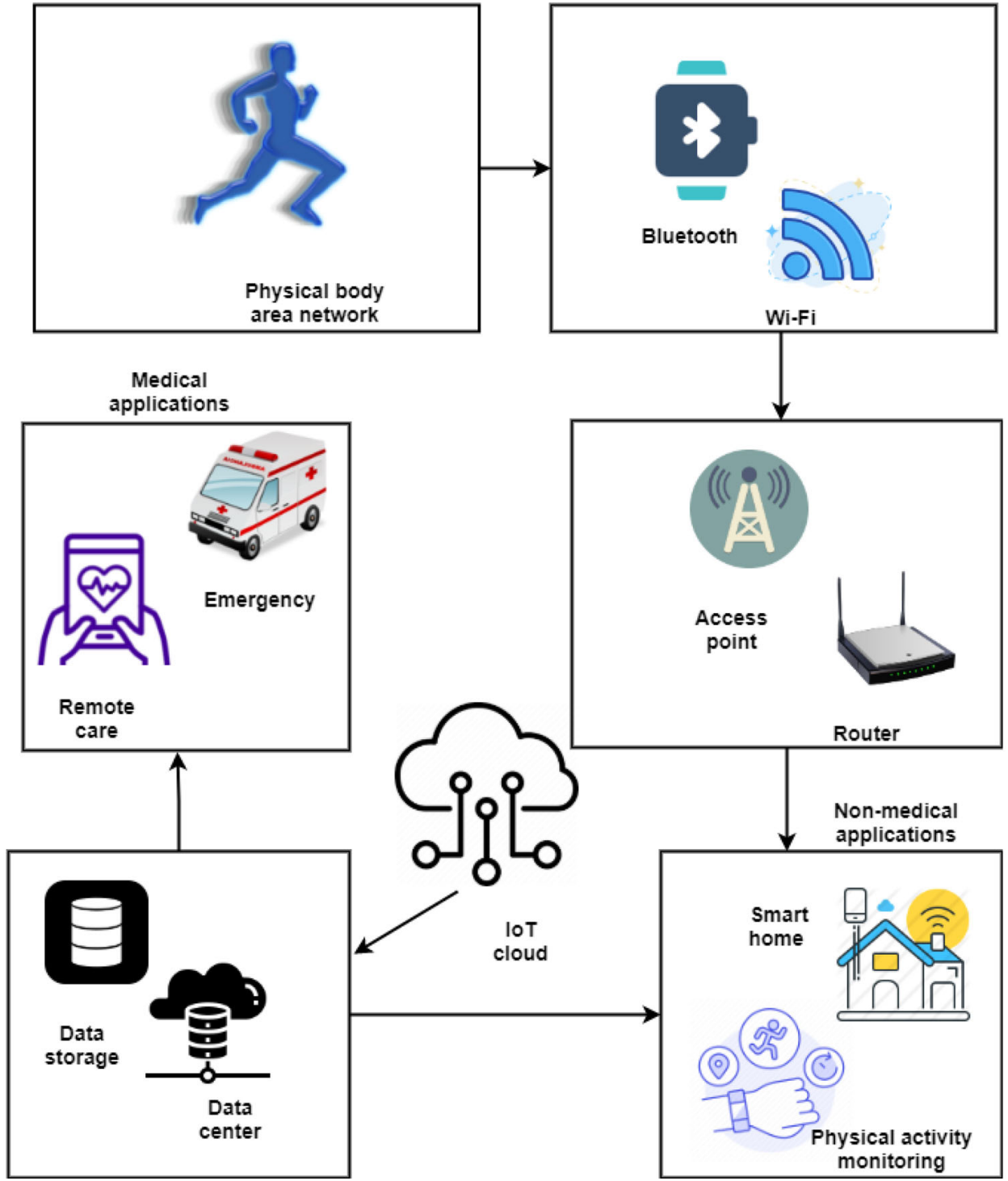

router that uses cellular WAN connections is a smart Internet gateway that IoT devices and sensors use to access the Internet. A hard-wired Internet connection such as Ethernet, fiber optics, cable, DSL, etc., is no longer required for the IoT system to work. The access points act as a hub that connects all stations. It acts as a focal point for communications, extending the range of wireless users' connections. The local area network provides a different place from which devices can join the network, allowing more devices. This paper aims at cleaning and degrading information organized into time windows that are the same and uneven to identify them. The main signal functionality is extracted in extraction phases to create a more flexible and powerful screen. Combining the student context leads to a high level of regular activity, such as eating, cooking and dressing.

Physical activity monitoring technique is widely used to monitor various the physical health condition that an individ- ual communicates possible abnormalities, reducing clinical times, reducing hospital costs, and improving quality of care in real time to students, parents, and the trainer. IoT applications gathering physical data from the student to be accessible remotely can monitor health care systems. The user interface, data collector, and Internet connectivity are part of those applications. The IoT device storage integration and data transfer architecture can be implemented in this approach. This approach aims to visualize sensed data in real time to transmit, store, and turn data accessible; development of IoT-based platform providers that integrate people healthcare providers and devices in the same context. It is an Internet data management platform that improves and standardizes IoT applications and addresses device interoperability. Healthcare professionals can evaluate student data from faraway locations and request emergency help if necessary. Another solution is a smart device portal that takes 
Fig. 3 Different types of wearable device technology

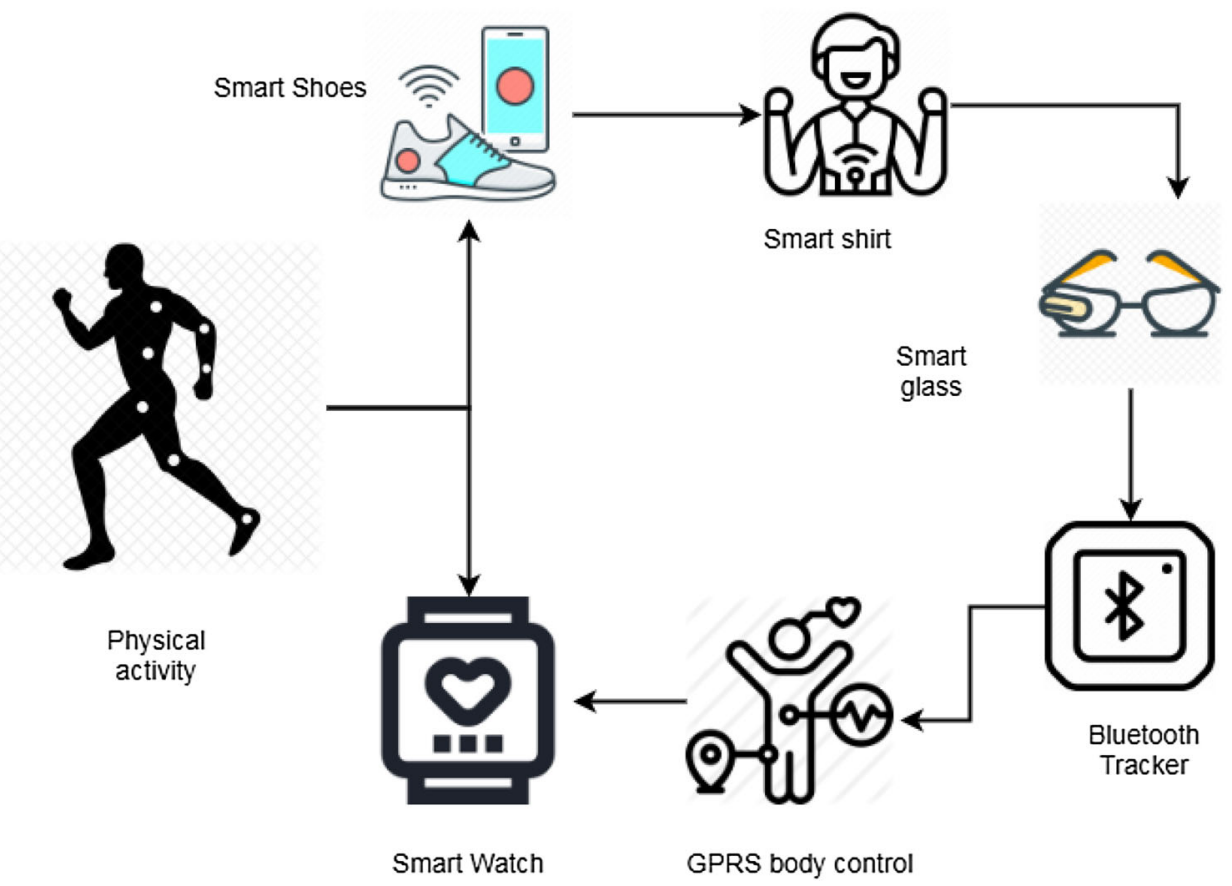

data processes and saves them in the IoT cloud for wearable device monitoring to every physical health system. Figure 2 illustrates a wireless network body architecture, a personal server using a smart home digital assistant, and a medical server on an IoT wearable device system. The supervisor system displays it and coherently by receiving preprocessed data. The results suggest that the quality of life of persons is improved. A device's hosting effectively shows the saved data on the website. Healthcare providers can easily access the Internet to evaluate the data. A physical activity monitoring system is proposed for body temperature. It offers non-invasive monitoring of blood glucose that sends student data in real time to healthcare professionals. The device proposed and monitored the physical condition of students using a monitoring system and a heart rate sensor.

The flexing angle data created by the physical activity are transmitted for wearable device analysis, preventing several times a week from traveling to the doctor. Healthcare providers transmit the acquired data to the cloud for IoT device access. A student physical health monitoring platform is described for data collections, and sensors are connected directly to the IoT device for student data. The data are processed and saved in the IoT cloud for healthcare providers to access and monitor the physical condition. The IoT technology allowed for personalized physical healthcare system implementation and success in health, considering the current work on physical healthcare systems enabled by IoT and technology capabilities for IoT health care. The monitoring device can identify participants who can benefit from a physical activity monitoring system once positive results. Integrated cloud computing and the IoT system for health surveillance of students and efficient virtual care surveillance system with heart disabilities using ECG are highly encouraging. A secure data aggregation management approach for physical health monitoring systems improves data collection quality and security. Security research shows that the suggested technique protects the confidentiality of data authenticity and data security can effectively survive passive scam and reproduction attacks.

Figure 3 shows the types of wearable device technology. Wearable devices, such as watches, shoes, shirts, glass, and body sensors, can be attached to the body, as illustrated in Fig. 3. These devices are supposed to connect them to student body sensors to show body temperature, heart rate, and blood pressure. A method designed for monitoring vital signs at rest homes for people is detailed in this connection. Student clothing sensors collect information to monitor their aspects of health. Suppose a student presses a button, and the important data send for analysis to the healthcare professionals, such as oxygen saturation, blood pressure, and heart rate. The research, as though the system presented, has more particular wearable devices. It allows access on the cloud to real-time data, and monitors blood pressure, temperature, and oxygen saturation. Data are collected using wearable devices and transmitted for analytical purposes to physicians. More generic systems are found that collect devices store and retrieve data in real time on the cloud. The Internet of things and medical devices as alternative technologies for identifying and controlling stress by cloud-based services, digital assistants, and wearable smart health devices for collecting and processing significant data sets that monitor long-term stress behavior. An ECG remote monitoring sys- 
tem integrated with the Internet of things for long-term health regulations. A study of the wearable device and the respective data integrity and privacy measures available once smart wearables are exposed to numerous cyber threats creates new security difficulties in collecting data and other connected IoT components and applications.

Wearable devices are designed for each person to implement their moving vectors in a virtualized context. These evaluate their localized positions with the main objective of walking a prediction system to increase detection performance. The advantages of wearable and Internet-of-things technologies in improving realistic task-based physical education situations show that using these solutions can be effective by freeing the trainers from recording each student's tasks during the classroom. Characteristics of IoT scenario wearable applications and interaction designs between wearable devices and smart objects are discussed. Implementation of an application for wearable devices to evaluate defined models of interactions in an intelligent setting employed in the IoT testing ground of their department. The wearable devices and IT infrastructure utilized for disease consider how this technological device might transform diagnostics and treating paradigms. A method for detecting and monitoring virus infections is based on physical health monitoring system IoT and cloud technologies.

Figure 4 shows the physical activity index for student data. Given the limited and real decision signal providing relevant data for the variation in the received power the physical activity monitoring method quantifies the physical activity, an index is used in the monitoring system. The proposed measure is the standardized power to demonstrate the student's training. The standard power ensures the independence of the physical activity index from a distance between the monitoring device and the student

$\mathrm{AI}(m)=\sum_{i=1}^{m_{a}} \max g_{c}\left(p_{m}(i, j)\right)$.

As Eq. (1) describes, combine the variation of the action according to several columns $i$ and $j$. For a demonstration of the activity index $(m)$, estimate in each column the sum of the standardized power. Where $M$ is the entire time recorded for $p_{m}$ represents a standardized health mapping power, and $\max g_{c}$ indicates a maximum column

$\mathrm{IMD}=\int_{p=p_{0}}^{p_{0}+P} b_{y}(p) \mathrm{d} t$.

As Eq. (2) defines, the extensive experiments show that students can accurately estimate energy consumption using a monitoring device and increase the proposed system $F$ score value. In the period $P$ starting at $p_{0}$, the estimation

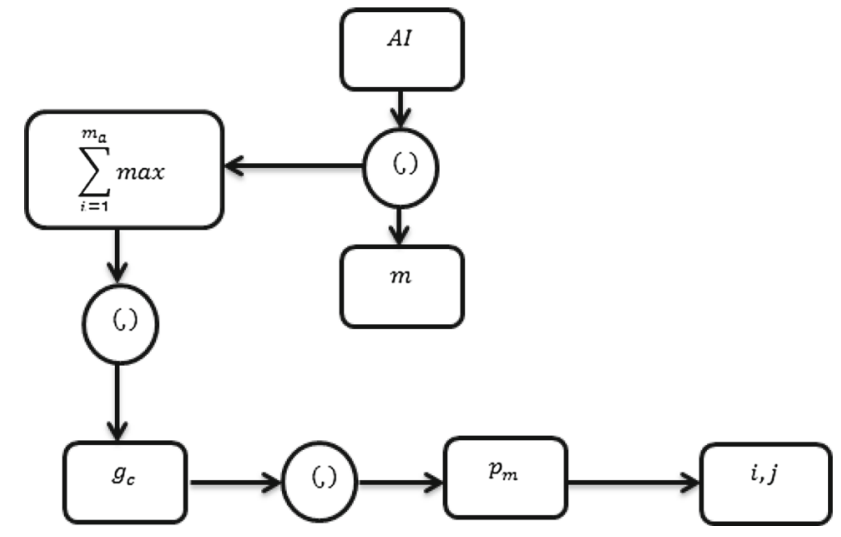

Fig. 4 Physical activity index

bases on a physical activity value calculated as follows in Eq. (2). This activity value of IMD, the integral part of the device output modulus, correlates with the signal energy of all accelerometer axis over the time $b_{y}$. The amount of movement experienced by the sensor during period $\mathrm{d} t$, therefore, matches well. Before integration, the signal is reduced to a bandwidth of between 0.11 and $20 \mathrm{~Hz}$ to remove frequency components (mainly gravity) that are not part of active physical activity.

The vector position $p_{j}$ measures the value of the di total between the two consecutive sample values of the $i$ th coefficient. It creates a straight line of two points and is defined as

$p_{j}=\sqrt{s_{j}+s_{j+1}+s_{j+2}}$.

As equation explores when the current three device signal samples are $s_{j}, s_{j+1}$ and $s_{j+2}$ as described in Eq. (3). The physical activities running and walking are shown with a red signal and a blue signal. The vector of the running activity is represented by a purple dotted line with a green dotted line representing the position vector of the walking activity

$\mathrm{MA}=\sum_{n=0}^{N} b_{j} g_{n+j}+\mu_{n}$.

As Eq. (4) computes, each activity signal is shown by the moving average MA function as a feature vector of the preceding sample plus the error sequence. These aspects describe the specific activity pattern $\mu_{n}$ and return an actual value when the pattern is found. It is used especially for feature extraction and is defined as Eq. (4). Here, $g_{n+j}$ is the sample data's $n+j$ feature $b_{j}$ indicates MA and the current sensor signal sample $n$ is an error series. For every activity pattern, the moving average used in this paper gives the maximum result.

Figure 5 shows the physical activity monitoring data from the wearable device. The waveform length features are used 


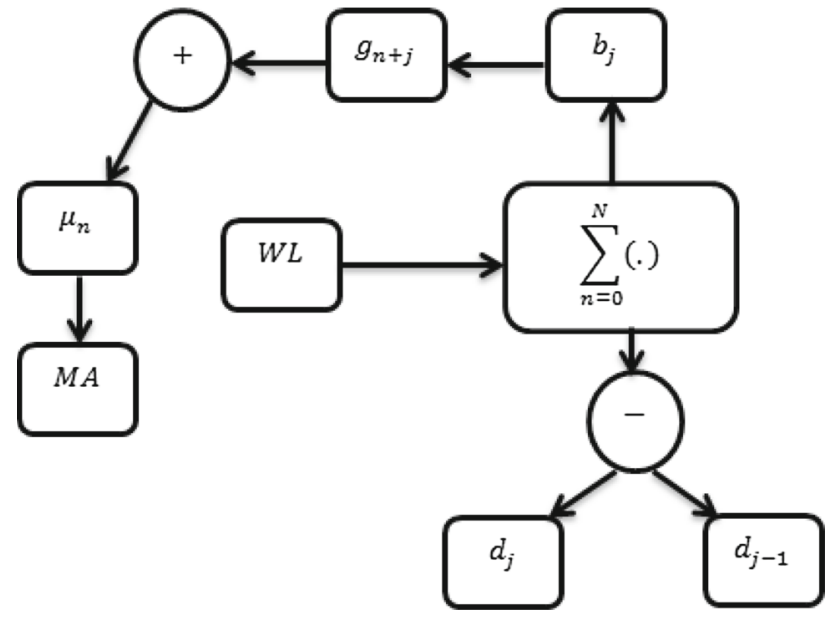

Fig. 5 Physical activity monitoring data

primarily for the measurement of the signal complexity in ECG data. These are used by wearable sensing devices to monitor human physical activity. In addition, by collecting the negatives of the current sample from the last sample and adding these, this equation effectively estimates each activity signal's complexity. These features provide information on amplitude, frequency, and time

$\mathrm{WL}=\sum_{n=0}^{N}\left(d_{j}-d_{j-1}\right)$

As Eq. (5) calculates here, waveform length WL is physical activity data and $d_{j}$ is the actual sample's feature vector, $d_{j-1}$ is the previous sample, and $N$ is the total sample number in the original activity signal.

The entire section summary can be demonstrated as follows: this section began with the theoretical background to the proposed IoT-assisted physical activity monitoring system and its working principle. The major component of physical and sports education was well deliberated. This section then presented the architectural design of the physical activity and resource management with smart care monitoring analysis. The statistical modeling of the proposed framework was finally detailed with mathematical formulations.

\section{Results and discussion}

A significant technical way to enhance the academic and effective level of physical education training in educational institutions can be to improve the creation of the level of education by virtual networks in educational institutions and actively support education reform. This paper explores the application prospects for the technology of wearable tech- nology in the institution of physical education. The system experiences a virtualization network and wearable device end collects required data and interacts in real time from the IoT. The IoT wearable devices can offer virtual technology to set up a real-time information system, scientific reference system, and advanced technologies platform. First, various physical activity monitoring devices are studied in IoT and identified in it. The solution provided for student health is based on the intelligent wearable device. It collects the heartbeat rate and the temperature level of the student and notifies trainers in real-time data access. In this work, the possibility of using IoT wearable devices sensed data as a resource for active monitoring had been established using a method of non-contact physical activity monitoring. The experimental results demonstrate that monitoring physical activity effectively collects student physical movements and activities in the health care application. Physical activity monitoring is designed for wearable devices that allow the continuous collection of student physical data. Some studies have shown that wearable devices that collect essential physical and physiological student data have increased. These aim to monitor the student's everyday physical activity at developing technology to monitor and support physical conditions, including heart rate, body temperature, and virtual and wearable device sector development. The order of representing the physical activity monitoring data of the simulation parameters of IoTPAMD is shown in Table 1.

\section{(i) Accuracy ratio (\%)}

The deep reinforcement system model improves accuracy and indicates significant changes in student physical activity. Instead of using a monitoring device for collecting data to classify the physical activity with high accuracy has increased and the average error is reduced using a multi-sensor device approach. The capacity to enhance categorization accuracy and estimate is associated with using a single sensor device by multiple devices and integrating data to provide more reliable information on physical activity in meditative decision-making. The physical activity dataset produces an accelerometer in various positions, such as the knee, chest, and wrist, combining monitoring devices like shoulder, ankle, chest, and wrist. For the three models, the accuracy is calculated: framework fusion, class fusion, and reverse fusion. The average accuracy of the proposed physical activity monitoring is indicated in Fig. 6. Explicitly connected functions can provide more relevant data to physical activity and lead to a loss of accuracy of assessment and classification. The changes in the accuracy of the physical state classification produced using data from the proposed device fusion have been evaluated. The results show that the suggested approach achieves excellent accu- 
Table 1 Student physical activity monitoring data using IoT-PAMD

\begin{tabular}{llllll}
\hline Parameters & PARMM & PEPLA & HARS & LTPA & IoT-PAMD \\
\hline Accuracy ratio (\%) & 58.3 & 64.4 & 73.1 & 82.6 & 98.3 \\
Prediction ratio (\%) & 55.1 & 65.5 & 71.2 & 83.7 & 96.5 \\
Interaction ratio (\%) & 56.2 & 66.3 & 75.4 & 86.5 & 95.4 \\
Efficiency ratio (\%) & 57.2 & 68.4 & 76.3 & 88.6 & 93.2 \\
Performance ratio (\%) & 59.4 & 70.5 & 78.4 & 88.9 & 95.1 \\
Error rate (\%) & 88.3 & 74.5 & 65.9 & 44.7 & 17.5 \\
$F$-score & 60.4 & 72.2 & 79.9 & 90.1 & 92.2 \\
\hline
\end{tabular}

performance. More study concerning the benefits of student physical education needs to be done until conclusions are established. The experimental study indicated that low-level students' academic performance in physical activity in education is significantly different. Trainers can use sports activity monitoring to improve the teams' performance, studying quickly how each player performs in the field and how their team is organized in total. Finally, the monitoring and classification of activity can automatically give performance data transfers and control during a match. The value of the performance evaluation cannot be overrated in this procedure. The physical education trainer and monitoring device can be used for physical activity evaluation. Since trainers recognize approximately half the essential sports activity events and it is perfect to use a motion sensor device for data reference to monitor all the actual activities. The physical and sports monitoring system should be available for physical trainers. Sports activity can use device regression to assess and develop student capabilities while preventing harm. Figure 7a shows performance ratio (\%) and Fig. 7b shows interaction ratio (\%).

The system is used to assess the physical activity, educational contexts, and interactions of the trainer, and this study combined features of an academic setting. The impression of training and the interaction between students is used for positive physical health. Associations for each result and attribute are studied for samples and linear regression. The student level of activity, lessons, and student interactions to development are recorded simultaneously. The average values of each vector have been obtained to evaluate how physical activity, educational setting, and student interaction are connected with training, college, and class characteristics. The analysis involved assessing the qualities of trainers, academics, and students in correlation with their physical activity, educational background, and interaction with educational institutions. The monitoring of student position previously depends on several methods of collecting data, from 


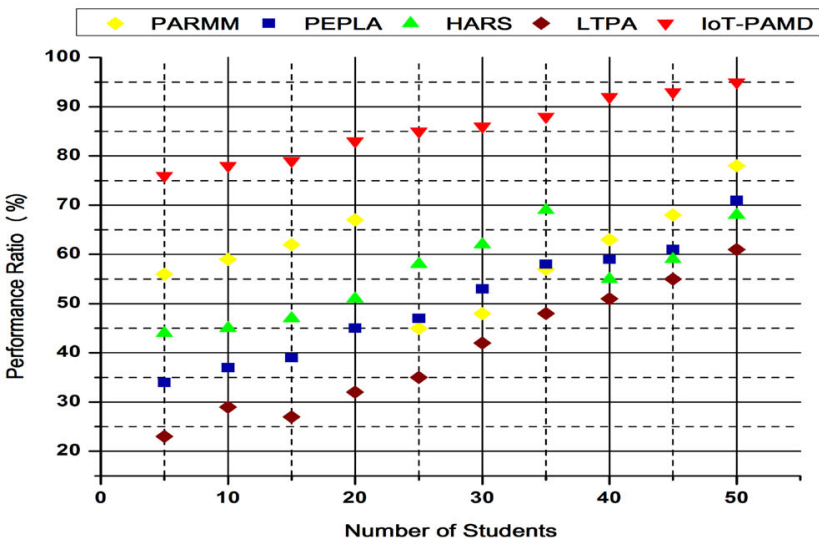

(a)

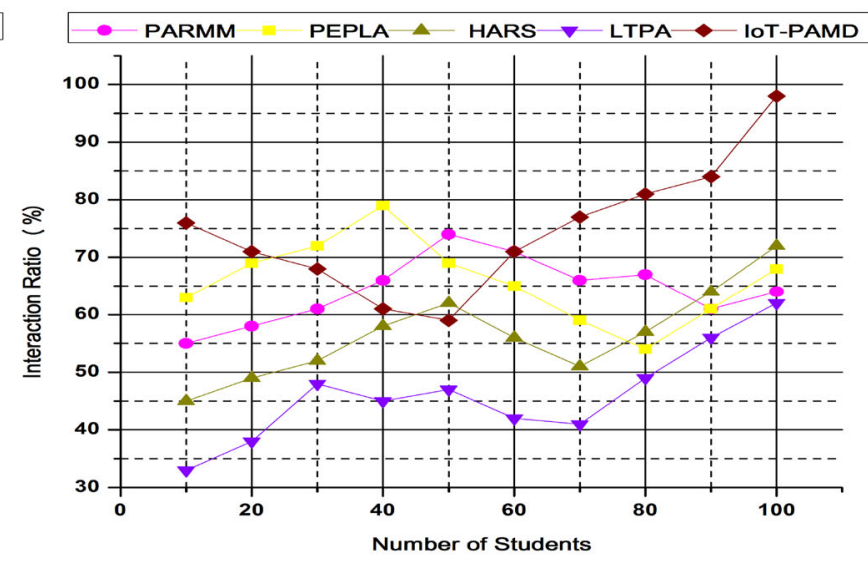

(b)

Fig. 7 Performance ratio (\%) and interaction ratio (\%)

monitoring the process to visual analysis. As it requires a lot of effort to manage and evaluate such data manually, the research concentrated mainly on a small number of students from several places. The application of auto-monitoring devices in team sports is limited by insufficient virtual reality technology on playgrounds. However, it is a huge challenge to resolve the complex nature of effort present in many physical activities. Sports students are recognized to adapt many conditions of their speed and flexibility. There are frequent and predictable interactions with other sports students. Good interaction is a part of many successful sports activities, and trainers who interact actively with students can influence effective communications directly, pay attention, and understand the opinions of others.

(iii) Prediction ratio (\%) and error rate (\%)

Physical activity training has an important role in improving basic abilities such as strength, speed, durability, flexibility, agility, strength, and balance. Students are vital to having good performance in numerous sports and physical activity. Physical education provides the framework for routine and structured physical activity participation. The curriculum's physical activity participation position is probably characterized by its focus on health and fitness in this aspect. The extent to which this explanation is accurate is uncertain and rarely examined. However, the assumption seems to be valid, because physical engagement usually plays a major role in students' daily physical activity. The significant function of physical education in promoting positive physical activity in national health objectives is illustrated. The improvements in the students engaging in everyday physical engagement activities are discussed in physical education. This paper analyzed the connections between student health rates, predicted behavior mediation conditions, and limitations. The data revealed a longer, reduced digital audio prediction, reduced sleeplessness, and enhanced mood among students with a larger degree of physical activity. In linear regression analyses, effective cognitive, physical state, and functioning are used to evaluate subjective and objective healthcare prediction duration of physical activity and student interactions. A variety of multiple regressions can additionally study the association between health and physical activities. The analysis shows that lower rates predict a self-reported trend and that students significantly reduce this effect. Figure $8 \mathrm{a}$ shows the prediction ratio (\%) and Fig. $8 \mathrm{~b}$ shows error rate $(\%)$.

This research evaluated probable interactions between student health rates in the institution, the limitations, and the circumstances for mediating in society. The data confirmed that students with increased physical activity predicted a more extended, reduced digital audio, reduced sleeplessness, and increased mood. In linear regression analysis, the physical activity and student interactions of the subjective and objective length of heath predictions are analyzed using impacted and cognitive, physical activity, and function. Several regressions aim to study better the associations between health and physical activity and socioeconomic class. The regression analyses indicate that fewer error rates suggest a trend toward self-reporting students are significantly lower active. The purpose of the present study is to determine that the student's educational outcomes are forecast for well-being, phases of relaxation, and mediation. Many studies study the collective prediction of the highest levels of quality, lower limits, and variables. The error rate for calculating a physical activ- 


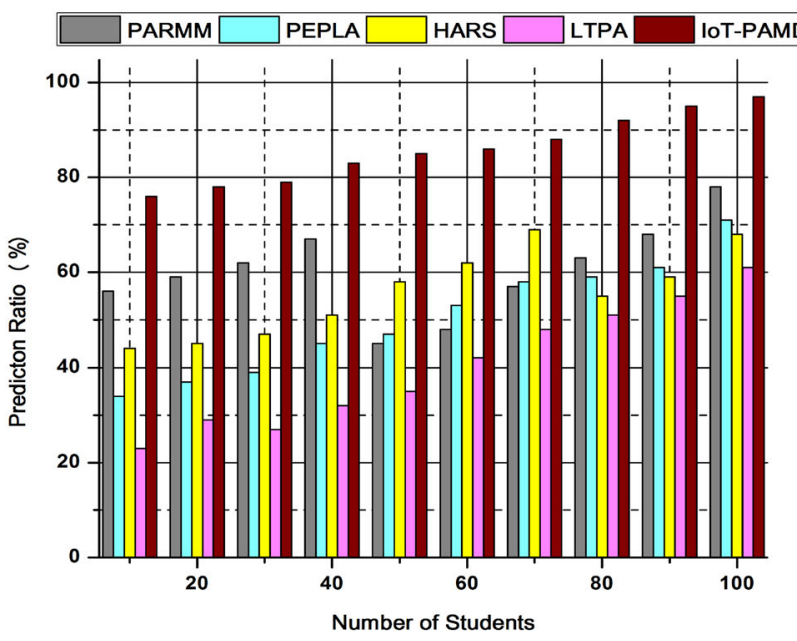

(a)

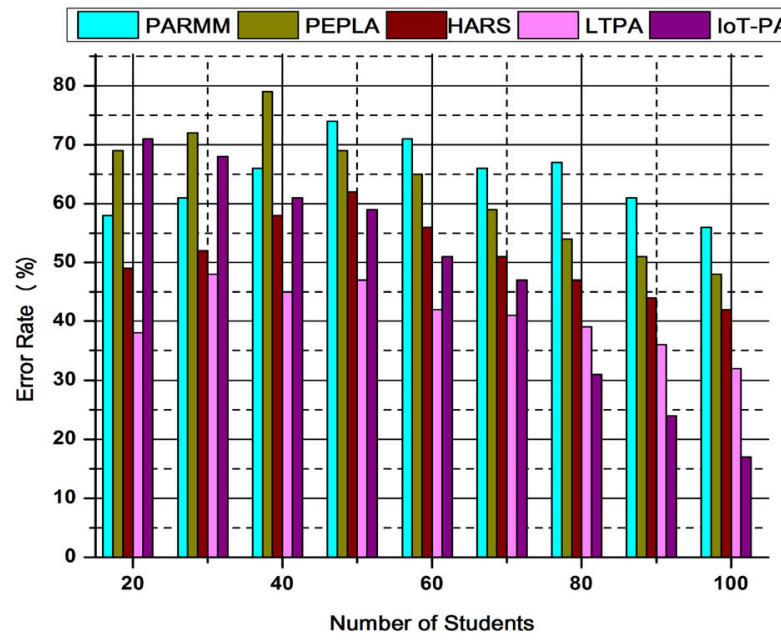

(b)

Fig. 8 Prediction ratio (\%) and error rate (\%)

ity assessment that uses physical activity can be seen in the ratio of overall energy spending to base energy spending to connect study-based estimates of physical activity levels with the real levels of lifestyle. The structure of the anticipated measurement error rate can apply to other institutions in this group. Students with a measuring error model developed small sections of connection and augmentation for physical activity.

iv) Efficiency ratio (\%)

The analysis of student physical health measures collected by the physical education monitoring of the highly efficient use of physical activity devices in the physical training of students. The excellent efficiency of the analytical scheme of modern physical activity innovation for student education is highlighted. Health facility processes monitoring and other health attributes improve the system's consistency and dependability and significantly reduce health and error rates. The results confirmed the analysis of the physical activity and the impact of sport on success measurements, physical education, health, performance, and career options in several studies. A similar approach to physical activity efficiency for students is the physical activity index, achieved using movement technologies. Among other aspects, it increases during routine training and reduces if training is weak and desirable. The focus is important in physical education and other fields, and attention intensity is key to effectively accessing information. Optimizing the physical training system includes highly efficient educational institutions and physical education, producing college of education training, encouraging trainers to create a new physical activity education system of abilities. In demanding and
Table 2 Efficiency ratio (\%)

\begin{tabular}{llllll}
\hline $\begin{array}{l}\text { Number of } \\
\text { students }\end{array}$ & PARMM & PEPLA & HARS & LTPA & IoT-PAMD \\
\hline 10 & 50.1 & 62.3 & 70.1 & 80.1 & 87.6 \\
20 & 52.3 & 62.4 & 71.2 & 80.5 & 88.3 \\
30 & 53.6 & 63.5 & 72.7 & 81.5 & 89.9 \\
40 & 54.5 & 64.5 & 73.8 & 82.6 & 90.4 \\
50 & 55.6 & 67.9 & 74.6 & 83.4 & 91.1 \\
60 & 56.8 & 68.3 & 75.7 & 84.5 & 92.7 \\
70 & 57.7 & 69.8 & 76.8 & 85.6 & 93.9 \\
80 & 58.9 & 69.9 & 79.5 & 85.9 & 94.1 \\
90 & 59.5 & 70.2 & 80.1 & 86.1 & 94.5 \\
100 & 60.2 & 70.9 & 80.9 & 86.9 & 93.2 \\
\hline
\end{tabular}

intensive training constitutionally, students with potential physical activity efficiency should obtain higher outcomes. Table 2 shows the efficiency ratio (\%).

(v) $\boldsymbol{F}$-score (\%)

The $F$-scoring assessment of physical activity using a dataset with such a sensing device and combinations of monitoring devices are shown in Table 3. The various actions proposed in the dataset are evaluated by the simulation tool such as walking, standing, sitting, lying, etc. For the knee, chest, and wrist and combinations of sensors, activities are generated, and the results are evaluated and reported. The results reveal that when devices are coupled, the performance of physical activity student improves engagement. For simulation analysis, the different positions of the device like the knee and wrist can be examined. This results in the combination of the performance of both sen- 
Table $3 F$-score (\%)

\begin{tabular}{llllll}
\hline $\begin{array}{l}\text { Number of } \\
\text { students }\end{array}$ & PARMM & PEPLA & HARS & LTPA & IoT-PAMD \\
\hline 10 & 52.3 & 66.7 & 76.0 & 80.3 & 89.5 \\
20 & 55.4 & 67.7 & 76.1 & 81.4 & 89.9 \\
30 & 59.0 & 68.9 & 77.2 & 82.5 & 85.1 \\
40 & 61.0 & 70.0 & 77.3 & 83.4 & 86.2 \\
50 & 64.8 & 71.1 & 77.7 & 85.1 & 87.5 \\
60 & 66.8 & 72.2 & 78.1 & 85.9 & 88.3 \\
70 & 67.7 & 73.2 & 78.5 & 86.3 & 89.3 \\
80 & 60.9 & 74.5 & 78.9 & 87.4 & 90.0 \\
90 & 60.5 & 75.6 & 79.3 & 88.5 & 91.3 \\
100 & 65.2 & 75.7 & 80.1 & 89.1 & 92.2 \\
\hline
\end{tabular}

sors, where physical activity has the highest $F$-score. For example, the device, for example, ankle, chest, and wrist, is used for analyzing student physical activity by combining their results, for example, ankle and wrist. The average $F$-score is calculated; the result is shown in the figures above. The results indicate that physical activity performs most and combines devices with physical activity predictions. Table 3 shows the $F$-score (\%).

The proposed system IoT-PAMD shows the high accuracy ratio, prediction ratio, interaction ratio, performance ratio, efficiency ratio, $F$-score, and reduced error rate when compared to physical activity recognition and monitoring model (PARMM), physical education and physical literacy approach (PEPLA), human activity recognition system (HARS), and leisure-time physical activity (LTPA) methods. Regarding wearable device technology and IoT concerns, this study reveals deep reinforcement learning in physical education that recognizes the importance of physical exercise.

\section{Conclusion}

This study finds deep reinforcement learning for the analysis of physical education that acknowledges the essential physical activity of wearable device technology concerning IoT concerns. Students with different physical and functional status levels have developed and analyzed an end-to-end framework for solving such difficulties. The data analysis key contribution is divided into two parts: monitoring various physical activities using IoT wearable devices in physical health students, including everyday walking, jogging, and sitting to practicing active people regularly. The challenges are met through the efforts required to provide the IoT wearable devices to monitor student physical activity with a performance ratio using IoT-PAMD solutions exam- ined. The experimental results prove the reliability of the proposed framework by improving the high accuracy ratio (98.3\%), prediction ratio (96.5\%), interaction ratio $(94.4 \%)$, performance ratio $(95.1 \%)$, the efficiency ratio $(93.2 \%), F$ score $(92.2 \%)$, and reduce error rate $(17.5 \%)$ compared to the other methods. Overall, the results justify the proposed strategy to identify the student's physical health for additional monitoring. In the future, the security system for the proposed model has been planned to integrate to improve performance and credibility.

Open Access This article is licensed under a Creative Commons Attribution 4.0 International License, which permits use, sharing, adaptation, distribution and reproduction in any medium or format, as long as you give appropriate credit to the original author(s) and the source, provide a link to the Creative Commons licence, and indicate if changes were made. The images or other third party material in this article are included in the article's Creative Commons licence, unless indicated otherwise in a credit line to the material. If material is not included in the article's Creative Commons licence and your intended use is not permitted by statutory regulation or exceeds the permitted use, you will need to obtain permission directly from the copyright holder. To view a copy of this licence, visit http://creativecomm ons.org/licenses/by/4.0/.

\section{References}

1. Sheron PF, Sridhar KP, Baskar S, Shakeel PM (2020) A decentralized scalable security framework for end-to-end authentication of future IoT communication. Trans Emerg Telecommun Technol 31(12):e3815

2. Shahriar MR, Al Sunny SN, Liu X, Leu MC, Hu L, Nguyen NT (2018) MTComm based virtualization and integration of physical machine operations with digital-twins in cyber-physical manufacturing cloud. In: 2018 5th IEEE international conference on cyber security and cloud computing (CSCloud)/2018 4th IEEE international conference on edge computing and scalable cloud (EdgeCom). IEEE, pp 46-51

3. Zhong CL (2020) Internet of things sensors assisted physical activity recognition and health monitoring of college students. Measurement 159:107774

4. Nguyen NT, Liu BH, Pham VT, Luo YS (2016) On maximizing the lifetime for data aggregation in wireless sensor networks using virtual data aggregation trees. Comput Netw 105:99-110

5. Thota C, Sundarasekar R, Manogaran G, Varatharajan R, Priyan MK (2018) Centralized fog computing security platform for IoT and cloud in healthcare system. In: Fog computing: breakthroughs in research and practice. IGI global, pp 365-378

6. Ranjan G, Nguyen TN, Mekky H, Zhang ZL (2020) On virtual id assignment in networks for high resilience routing: a theoretical framework. In: GLOBECOM 2020-2020 IEEE global communications conference. IEEE, pp 1-6

7. Wang Y, Muthu B, Sivaparthipan CB (2021) Internet of things driven physical activity recognition system for physical education. Microprocess Microsyst 81:103723

8. Nguyen TG, Phan TV, Hoang DT, Nguyen TN, So-In C (2020) Efficient SDN-based traffic monitoring in IoT networks with double deep Q-network. In: International conference on computational data and social networks. Springer, Cham, pp 26-38 
9. Vangala A, Das AK, Kumar N, Alazab M (2020) Smart secure sensing for IoT-based agriculture: blockchain perspective. IEEE Sensors J 21(16)

10. Manogaran G, Baabdullah T, Rawat DB, Shakeel PM (2021) AI assisted service virtualization and flow management framework for $6 \mathrm{G}$-enabled cloud-software-defined network based IoT. IEEE Internet Things $\mathrm{J}$

11. Arshad J, Azad MA, Amad R, Salah K, Alazab M, Iqbal R (2020) A review of performance, energy and privacy of intrusion detection systems for IoT. Electronics 9(4):629

12. Cheng J (2021) Evaluation of physical education teaching based on web embedded system and virtual reality. Microprocess Microsyst 83:103980

13. Manogaran G, Shakeel PM, Fouad H, Nam Y, Baskar S, Chilamkurti N, Sundarasekar R (2019) Wearable IoT smart-log patch: an edge computing-based Bayesian deep learning network system for multi access physical monitoring system. Sensors 19(13):3030

14. Gao J, Wang H, Shen H (2020) Machine learning based workload prediction in cloud computing. In: 202029 th international conference on computer communications and networks (ICCCN). IEEE, pp 1-9

15. Sun Z, Kadry SN, Krishnamoorthy S (2021) Internet of things assisted advanced dynamic information processing system for physical education system. Technol Health Care 29(6):1263-1275. https://doi.org/10.3233/THC-213005

16. Gheisari M, Najafabadi HE, Alzubi JA, Gao J, Wang G, Abbasi AA, Castiglione A (2021) OBPP: an ontology-based framework for privacy-preserving in IoT-based smart city. Future Gener Comput Syst 123:1-13

17. Sivaparthipan CB, Muthu BA, Manogaran G, Maram B, Sundarasekar R, Krishnamoorthy S, Chandran K (2020) Innovative and efficient method of robotics for helping the Parkinson's disease patient using IoT in big data analytics. Trans Emerg Telecommun Technol 31(12):e3838

18. Kang TC, Wen CH, Guo SW, Chang WY, Chang CL (2020) The implementation of an IoT-based exercise improvement system. J Supercomput 76(8):6361-6375

19. Ahmad M, Ishtiaq A, Habib MA, Ahmed SH (2019) A review of internet of things (IoT) connectivity techniques. Recent Trends Adv Wirel IoT Enabled Netw 25-36

20. Wang Z, Liu D, Jolfaei A (2020) Resource allocation solution for sensor networks using improved chaotic firefly algorithm in IoT environment. Comput Commun 156:91-100

21. Abou-Nassar EM, Iliyasu AM, El-Kafrawy PM, Song OY, Bashir AK, Abd El-Latif AA (2020) DITrust chain: towards blockchainbased trust models for sustainable healthcare IoT systems. IEEE Access 8:111223-111238
22. Gad R, Abd El-Latif AA, Elseuofi S, Ibrahim HM, Elmezain M, Said W (2019) IoT security based on iris verification using multialgorithm feature level fusion scheme. In: 2019 2nd international conference on computer applications and information security (ICCAIS). IEEE, pp 1-6

23. Lei T, Cai Z, Hua L (2021) 5G-oriented IoT coverage enhancement and physical education resource management. Microprocess Microsyst 80:103346

24. Aicha AN, Englebienne G, Kröse B (2018) Continuous measuring of the indoor walking speed of older adults living alone. J Ambient Intell Humaniz Comput 9(3):589-599

25. Du J, Nan Z (2021) Research on the intelligent model of progress in physical education training based on motion sensor. Microprocess Microsyst 82:103903

26. Haible S, Volk C, Demetriou Y, Höner O, Thiel A, Sudeck G (2020) Physical activity-related health competence, physical activity, and physical fitness: analysis of control competence for the self-directed exercise of adolescents. Int J Environ Res Public Health 17(1):39

27. Telford RM, Olive LS, Keegan RJ, Keegan S, Telford RD (2021) Teacher and school outcomes of the physical education and physical literacy (PEPL) approach: a pragmatic cluster randomised controlled trial of a multicomponent intervention to improve physical literacy in primary schools. Phys Educ Sport Pedagog 26(1):79-96

28. Jalal A, Batool M, Kim K (2020) Stochastic recognition of physical activity and healthcare using tri-axial inertial wearable sensors. Appl Sci 10(20):7122

29. Lee S, Lee C, An J (2020) Psycho-social correlates of leisuretime physical activity (LTPA) among older adults: a multivariate analysis. Eur Rev Aging Phys Act 17(1):1-7

30. Zhang QQ (2021) Intelligent control model of athletes' training intensity based on big data analysis of physical fitness. In: Journal of physics: conference series, vol 1883(1). IOP Publishing, pp 012147

Publisher's Note Springer Nature remains neutral with regard to jurisdictional claims in published maps and institutional affiliations. 